$$
\text { DOE/ER/45280--T4 }
$$

\title{
X-RAY SCATTERING STUDIES OF NON-EQUILIBRIUM \\ ORDERING PROCESSES
}

\author{
Progress Report
}

DOE/ER/ 45280--T4

DE92 014006

November 1, 1989 - October 31, 1992

S. E. Nagler

Department of Physics, University of Florida

Gainesville, FL 32611

Prepared for the U. S. Department of Energy

Under Grant Number DE-FG05-91ER45280

\section{DISCLAIMER}

This report was prepared as an account of work sponsored by an agency of the United States States Government nor any agency thereof, nor any of their Government. Neither the United States Govers implied, or assumes any legal liability or responsiemployees, makes any warranty, express or implied, of anu information, apparatus, product, or bility for the accuracy, completeness, or usefulness of any inge privately owned rights. Referprocess disclosed, or represents that its use would not infringe price by trade name, trademark, ence herein to any specific commercial product, process, or ser imply its endorsement, recommanufacturer, or otherwise does not necessarily constiute or imply its endorereof. The views mendation, or favoring by the United States Government or any ate or reflect those of the and opinions of authors expressed herein do not

\section{MASTER}




\section{$\underline{\text { Abstract }}$}

This report summarizes the major results obtained under US DOE Grant Number FG05-90ER45280 from the dates November 1, 1989 through October 31, 1992 inclusive. The principal work includes $\mathbf{x}$-ray scattering studies of phase transition kinetics in binary alloy order-disorder transitions, block co-polymer crystallization, and charge density wave solids under applied electric fields, and studies of magnetic excitations in low dimensional quantum systems. 
$\underline{\mathrm{X}-R a y} \underline{\text { Scattering Studies of }} \underline{\text { Non-Equilibrium }} \underline{\text { Ordering Processes }}$

Table of Contents

I. Introduction

II. Research Activities

III. Invited Lectures

IV. Conference Presentations

V. Publications

VI. Travel

VII. Personnel

VIII. External Support

IX. Figures
Page Numbers

4-5

$5-11$

$11-12$

12-13

13-16

16

17

18

18-19 


\section{Introduction.}

The research performed under the auspices of this grant centers thematically on the role of time dependent changes in the degree of order in a system. The techniques used are $x$-ray scattering, which provides a direct measure of microscopic structure, and neutron scattering, which can also measure elementary excitations.

The major part of the work done has been thematically tied to the general problem of phase transition kinetics. The precise nature of the approach to equilibrium from a metastable or unstable state is one of the forefront questions in statistical physics. It is also an important question for technology, inasmuch as the properties of materials can be drastically altered by varying the conditions of preparation and processing. The work reported on here concentrates on real materials that are in some measure chosen as relatively simple examples so that one may hope to elucidate the underlying physics of the general problem. Materials that have been examined include metallic binary alloys, block co-polymers, and charge density wave materials. In each case the static equilibrium properties of the materials are carefully studied initially, followed by non-equilibrium experiments in which external parameters such as temperature or field are quickly varied (usually through a first order phase transition) resulting in a system that is initially far from equilibrium. These studies involve time-dependent scattering, which is essentially a diffraction "movie" of the evolution of long range order and microscopic structure. The details of the work are described in the next section.

More recently some studies of excitations in low dimensicnal magnetic systens have been carried out. The quantum mechanical nature of the excitations is particularly interesting scientifically, and the current understanding involves concepts from both field theory and traditional condensed matter physics. An understanding of these systems may also be important for other related problems including the mechanism for high $\mathrm{Tc}$ 
superconductivity and the problem of highly correlated electrons. Our research primarily involves neutron scattering studies of model materials in the extreme quantum limit as described in the next section.

The rest of this report is organized as follows: Section II describes the principal results obtained from this research, organized according to topic. Sections III through VIII contain information concerning presertations of this work, travel, and personnel relevant to the covered time period. Figures are in Section IX.

\section{II. $\quad$ Research Activities.}

A. Introduction

Several problems related to phase transition kinetics have been investigated experimentally. Systems studied include the $\mathrm{Cu}_{3} \mathrm{Au}$ alloy, diblock copolymers, blue bronze and others. Research has also been carried out on low dimensional magnetic systems. The progress and results obtained are summarized below, organized by topic.

\section{B. Order-Disorder Transition in $\underline{\mathrm{Cu}_{3} \mathrm{Au}}$}

The binary alloy $\mathrm{Cu}_{3} \mathrm{Au}$ is the protopypical example of an alloy with a first order "order-disorder" type phase transition. We have carried out several in-situ time-resolved $\mathrm{x}$-ray scattering studies on single-crystals and epitaxially grown films, utilizing both inl-house and synchrotron facilities (NSLS X-20A, X20C). Our experiments established several important results:

1. The lineshape of superlattice peaks is seen to change with time after a quench, reflecting the crossover to the late time scaling regime. This behaviour has been observed in totally different systems, and appears to be general. 
2. At late times the system demonstrates anisotropic nonequilbrium scaling, with the form of $S(Q, t)$ determined by the type of domain walls allowed in the system.

3. At intermediate times short lived metastable structures appear.

4. In thin films, there is a crossover to slower ordering, reflecting the constraints of dimensionality.

These results are described in publications $\mathrm{A10}, \mathrm{B} 2, \mathrm{C} 1, \mathrm{C} 3, \mathrm{C} 5, \mathrm{C} 7, \mathrm{D} 2$, and D3 listed in section $\mathrm{V}$ of this report.

The $\mathrm{Cu}_{3} \mathrm{Au}$ alloy has also been the subject of the first studies utilizing coherence properties of synchrotron radiation as described immediately below.

\section{Coherent X-Ray Diffraction}

The $\mathrm{x}$-rays produced by wigglers at NSLS (eg. beamline X-25) and those that will be produced at the APS have transverse coherence length that are typically several microns. This means that $\mathrm{x}$-ray beams collimated by pinholes of roughly 1 micron size or smaller will essentially be coherent, and hence show properties such as Fraunhofer diffraction which are not usually observed with $x$-rays. One of the consequences is that the scattering from a specimen shows the "speckled" intensity pattern more commonly seen with light scattering from coherent sources such as lasers. The first experiments to observe speckle in an $x$-ray diffraction pattern were performed at NSLS beamline X-25, and are described in publication B4 in this report.

In practice, the ability to measure speckle may be quite important for future work on nonequilibrium systems. The speckle arises from collective interference of scattered radiation originating from the entire illuminated volume of the sample. Using a time series 
of speckled diffraction patterns one may, in principle, measure time autocorrelation functions of the scattering intensity ("Intensity fluctuation spectroscopy). In a system where the degree of order is changing, the power spectrum of the autocorrelation functions is related to the microscopic flucuations ultimately responsible for the growth. To this end, time resolved coherent scattering experiments were carried out at NSLS X-25 on $\mathrm{Cu}_{3} \mathrm{Au}$ and $\mathrm{Fe}_{3} \mathrm{Al}$ crystals. (The experiments were carried out in March 1992 and the results are still being analysed). Some typical results are shown in Figure 1.

\section{Crystallization in Block Copolymers}

A thorough small angle $x$-ray scattering investigation of microphase separation and crystallization in the model diblock copolymer polystyrene-polybutadiene (PS-PB) was carried out. The time resolved experiments represent (to our knowledge) the first experiments of this type on polymer systems. The principal results are:

1. The structure at fixed density progresses from disordered (high temperature) to micellar fluid (narrow range of intermediate temperature) to a coexistence of micellar fluid and solid.

2. The crystalline structure is BCC, not simple cubic as previously reported in the literature.

3. After quenching, the development of the crystalline volume fraction with time does not obey the usual Avrami equation.

4. It appears (subject to confirmation) that oscillatory behaviour may be seen in the kinetics for shallow quenches.

The principal experimental results are described in publications $\mathrm{A} 2, \mathrm{~B} 1, \mathrm{C} 2, \mathrm{C} 6, \mathrm{D} 4$, and D5. 
As a result of the experiments, a new phenomenological theory was formulated to account for nucleation and growth in a coexistence region, described i publications A5 and C9.

\section{E. Blue Bronze}

Blue bronze $\left(\mathrm{KO}_{3} \mathrm{MoO}_{3}\right)$ is well known as a quasi-one dimensional charge density wave system. Much work has been done to characterize the nonlinear electronic transport properties of the material, and the CDW structure as a function of temperature and field. We have undertaken a study using high resolution and time resolved $\mathbf{x}$-ray scattering, and simultaneous in-situ transport measurements, with the ultimate goal of understanding the relationship between structure, transport, and defects in the system. Our experiments to date have been in-house, and synchrotron experiments will be performed at NSLS X-20 in June, 1992.

Our preliminary experiments have yielded some surprising results. Previous work showed that upon application of an electric field along the one-dimensional axis superlattice peaks characteristic of the CDW broadened in a direction perpendicular to the field. This has been explained as a consequence of the field inhomogeneities arising from uneven electrical contacts applied to the sample. Our work shows that, in fact the broadening is actually a splitting of the peak into two components, and repetitions on several different samples as well as altered electrical contacts on the aame sample indicate that it is an intrinsic feature. Our current thought is that the structure probably arises from pinning of the CDW's in a fashion that causes the phase to vary linearly with distance from a pinning site. Some typical results, including time resolved field switching data, are shown in figure 2. Preliminary work was described in publication C8, and preprint D1. 


\section{F. Other Work On Kinetics}

More work on kinetics is still in progress. To examine possible quantum effects on spinodal decomposition we have chosen to study phase separation in solid ${ }^{3} \mathrm{He} /{ }^{4} \mathrm{He}$ mixtures using small angle neutron scattering. To do the experiment requires the ability to rapidly change pressure in a sample cell mounted in a dilution refrigerator. We have constructed (at UF) a sample cell and corresponding gas handling system to be mounted on the neutron scattering compatible dilution refrigerator at ORNL. The current hold up is that the ORNL dilution refrigerator is in need of maintenance, but this is expected to be done by late summer.

Additional $x$-ray work is being carried out on crystallization in some elemental metals ( $\mathrm{Ga}, \mathrm{In})$ and ordering kinetics in $\mathrm{Mg}_{3} \mathrm{In}$. Some work on ferroelastic martensites (Au49\%Cd) has been carried out but requires completion.

\section{G. Low Dimensional Magnetism and Magnetic Excitations}

As the dimensionality of a magnetic system is lowered both quantum effects and thermal fluctuations become more important. One-dimensional systems are the extreme limit, and quantum effects which may be subtle (although possibly imporrtant) in higher dimensions become dominant. The one dimensional heisenberg antiferromagnet (1D HAF) is an important model many body problem, and also has many close realizations in nature. Inelastic neutron scattering investigations have been carried out on the quasi-one dimensional material $\mathrm{KCuF}_{3}$, which behaves like a $1 \mathrm{D}$ HAF with spin quantum number $\mathrm{S}=1 / 2$. The excitation spectrum of the system does not consist of a simple spin wave dispersion relation. The dynamical correlation function $S(Q, \omega)$, which is measured with neutron scattering, shows square-root singularities and a continuum scattering in energy at each wave-vector $Q$. 
Our experiments, which were among the first ever to exploit pulsed neutrons for measuring low dimensional excitations, clearly established the presence and form of the contunuum scattering, a purely quantum effect. (See figure 3) The experiments were initiated while S. Nagler visited Oxford university in the United Kingdom, and so far have been performed using the MARI chopper spectrometer at the Rutherford Appleton Laboratories. The experiments demonstrated that for sufficiently large characteristic energies the chopper spectrometer is a superb instrument for investigating 1 dimensional physics. Assuming that the PHAROS chopper spectrometer is commissioned at LANSCE, future pulsed measurements may be made there. In the meantime, triple axis experiments are planned at ORNL to examine the details of the long wavelength portion of the spectrum. The results of the experiments to date have appeared in publications A6, A9, and D6.

In addition we have carried out ultra-low temperature measurements on the model $\mathrm{S}=1$ quasi 1D HAF material NENP, in collaboration with the UF microKelvin group. At very low temperatures, the susceptibility of the material begins to increase. It is not yet known whether this arises from exotic end chain effects or the possible onset of incipient long range order (publications A11, C10).

Some other neutron scattering investigations of excitations in the 2D material $\mathrm{KFeF}_{4}$ (Publication A7) and the spin flop phase of $\mathrm{RbMnF}_{3}$ (Publication A8) were also carried out)

\section{H. Magnetic $\underline{X-r a y} \underline{S}$ attering}

Work was also initiated on magnetic X-Ray scattering. A preliminary experiment was carried out on $\mathrm{UPd}_{3}$. Experiments on the $5 \mathrm{~d}$ electron material $\mathrm{K}_{2} \operatorname{Re} S \ell_{6}$ are planned for the fall. 


\section{Other Related Work}

Much of the experimental work in this proposal utilizes $x$-ray synchrotron radiation. S. Nagler is a founding member on the executive board of MR-CAT, a proposed CAR for the Advanced Photon Source. The other members are B.A. Bunker (Notre Dame University), P. Dutta (Northwestern University), T. Morrison (Illinois Institute of Technology) and J. Faber (Amoco Research). MR-CAT has been approved by the APS proposal evaluation board and we plan to develop a full sector including an undulator beamline and bending magnet beamline.

\section{Invited Lectures.}

A. S.E. Nagler:

$\underline{1992}$

APS March Meeting Indianapolis, IN invited tutorial.

Steinhatchee Meeting on Condensed Matter Physics, Steinhatchee, FL.

University of Toronto, Toronto, Ontario, Physics Colloquium.

McMaster University, Hamilton, Ontario, Physics Colloquium.

University of Florida, Gainesvilie, FL, Physics Colloquium.

$\underline{1991}$

European Research Symposium on Dynamical Properties of Solids, Autrans, France.

University of Missouri, Columbia, MO, O.M. Stewart Colloquium.

MURR, Columbia Missouri, Seminar.

Rutherford Appleton Laboratories, UK, seminar.

Keele University, Stoke-on-Trent, UK, Physics Colloquium.

Institut Laue-Langevin, Grenuble, France, Seminar.

University of Chicago, Chicago, IL, James Franck Institute Colloquium. 
$\underline{1990}$

APS March Meeting, Anaheim, CA, invited symposium.

DOE/DMS Discussion Meeting on Neutron Scattering, Gatlinburg, TN.

Brookhaven National Laboratories, Upton, NY, NSLS seminar.

Oxford University, Oxford UK, Clarendon Laboratories seminar.

$\underline{1989}$

Ohio State University, Columbus, OH, Physics Colloquium.

\section{B. Other Personnel}

Several invited seminars on DOE research supported by this award were given by Dr. R.F. Shannon, Jr. and Dr. M.A. Sinch, most notably, and invited talk by Dr. Singh at the APS 1991 March meeting, Cincinnati, $\mathrm{OH}$

\section{Conference Presentations.}

$\underline{1992}$

APS March Meeting, Indianapolis, IN (3 presentations + invited tutorial).

Steinhatchee Meeting on Condensed Matter Physics, Steinhatchee, FL (invited).

$\underline{1991}$

European Research Symposium, Grenoble, France, (invited).

Amoco-University Poster Session, Naperville, IL.

International Conference on Magnetism, Edinburgh, UK, (selected talk). International Conference on Neutron Scattering, Oxford, UK.

APS March Meeting, Cincinnati, OH (1) (plus invited talk by M.A. Singh on DOE supported work). 
1990

APS March Meeting, Anaheim, CA, (invited plus 2).

DOE/DMS Discussion Meeting on Neutron Scattering, Gatlinburgh, TN.

$\underline{1989}$

APS March Meeting, St. Iouis, MO, (2).

StatPhys 17, Rio de Janiero, Brazil (selected talk).

\section{Publications.}

A. Refereed Journal Articles published or in press.

1. M.A. Singh, S.L. Smith, S.E. Nagler and W.J.L. Buyers "Low Temperature X-ray Study of UPd," Sol. State Comm. 74, 439 (1990).

2. C.R. Harkless, M.A. Singh, S.E. Nagler, G.B. Stephenson and J.L. Jordan-Sweet Small Angle X-ray Sicattering Study of Ordering Kinetics in a Block Copolymer Phys. Rev. Lett. $\underline{64}, 2285$ (1990).

3. J.H. Barry and S.E. Nagler, "Exact Solution for the Inelastic Neutron Scattering from an Anisotropic Honeycomb Magnet", J. Phys. Condensed Matter $\underline{3}, 3959$ (1991).

4. M. Sutton, S.G.J. Mochrie, T. Greytak, S.E. Nagler, L.E. Berman, G.A. Held and G.B. Stephenson "Observation of Speckle by Diffraction with Coherent X-rays", Nature $\underline{352}, 608$ (1991).

5. E. Domany and S.E. Nagler "Nucleation and Growth in the Coexistence Region", Physica A177, 301 (1991).

6. S.E. Nagler, D.A. Tennant, R.A. Cowley, T.G. Perring, and S.K. Satija "Spin Dynamics in the Quantum Antiferromagnetic Chain Compound $\mathrm{KCuF}_{3}$ ", Phys. Rev. B44, (1991). 
7. S. Fulton, S.E. Nagler, R.A. Cowley and L.M. Needham, "Spin-Waves in the Quasi-Two Dimensional Antiferromagnet KFeF\&", Physica, (1992).

8. D.A. Tennant, D.F. McMorrow, S.E. Nagler, R.A. Cowley and B. Fak, "Spin Wayes in the Spin Flop Phase of RbMnF ${ }_{3}^{\prime \prime}$, J. Mag. Mag. Mat., 104-107, 1079, (1992).

9. S.E. Nagler, D.A. Tennant, R.A. Cowley, S.K. Satija, T.G. Perring, A.D. Taylor and M. Arai, "Neutron Scattering Study of Quantum Excitations $\underline{\text { in }} \underline{\underline{a}} \underline{\underline{S}=1 / 2}$ Heisenberg Antiferromagnetic Chain", J. Mag. Mag. Mat., 104-107, 847, (1992).

10. R.F. Shannon Jr., S.E. Nagler, C.R. Harkless and R.M. Nicklow, "Time-Resolved

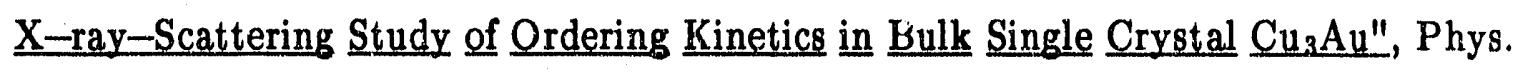
Rev. B. (to appear July 1, 1992).

11. O. Avenel et.al., "Low Temperature Magnetic Measurements of an $\underline{\mathrm{S}=1}$ Linear-Chain Heisenberg Antiferromagnet", Siubristted to Phys. Rev. Lett.

B. Ph.D. Dissertations

1. C.R. Harkless "X-Ray Scattering Study of Ordering Kinetics in Block Co-polymers", University of Florida, 1990.

2. R.F. Shannon, Jr. "A Time-Resolved X-Ray Scattering Study of the Ordering Kinetics $\underline{\text { in }} \mathrm{Cu}_{3} \mathrm{Au}^{\prime \prime}$, University of Florida, 1990.

C. Other Publications

1. R.F. Shannon, Jr., C.R. Harkless, M.A. Singh, S.E. Nagler, J.A. Dura, and C.P. Flynn, "Ordering Kinetics in Epitaxial Thin Films of $\mathrm{Cu}_{3} \mathrm{Au}^{\prime \prime}$, Bull. Am. Phys. Soc. $\underline{34}, 491$ (1989).

2. C.R. Harkless, M.A. Singh, and S.E. Nagler, "Kinetics of Microphase Separation Ordering in the Styrene-Butadiene Triblock Copolymer", Bull. Am. Phys. Soc. $\underline{34}$, 491 (1989). 
3. S.E. Nagler, R.F. Shannon Jr., C.R. Harkless, M.A. Singh, J.A. Dura and C.P. Flynn "Kinetics of Ordering in EpitaxialC113Au Films", in programs of the 17th IUPAP International Conference on Thermo dynamics and Statistical Mechanics, Rio de Janereo, (1989).

4. J.H. Barry and S.E. Nagler, "Exact Solution for $\underline{\operatorname{Sx}(q . \omega)}$ of Anisotropic Ising Model on Honeycomb Lattice" Bull. Am. Phys. Soc. 포, 553 (1990).

5. S.E. Nagler "Kinetics of orderi $\_g$ in Binary Alloys" Bull. Am. Phys. Soc. $\underline{\mathbf{3}}, 661$ (1990).

6. C.R. Harkless, M.A. Singh, S.E. Nagler, G.B. Stephenson and J.L. Jordan-Sweet "The Kinetics of Microdomain Ordering in the Styrene Butadiene Diblock Co-polymer" Bull. Am. Phyi. Soc. 포, 668 (1990).

7. R.F. Shannon, Jr., C.R. Harkless, S.E. Nagler, G.B. Stephenson and J.L. Jordan-Sweet, "Intermediate Stage Kinetics of an Order-n' - 2 rder Transition", Bull. Am. Phys. Soc. $\underline{36}, 379$ (1991).

8. J. Zhang, J.F. Ma, S.E. Nagler and S.E. Brown, "The Effect of Electric Fields on the Ordering of CDW at Low Temperatures", Bull. Am. Phys. Soc., 37, 110, (1992).

9. S.E. Nagler and E. Domany, "Nucleation and Growth in the Coexistence Region", Bull. Am. Phys. Soc., 37, 310, (1992).

10. J. Xu et.al., "Magnetic Measurements of a $\underline{1-D}$ Heisenberg Antiferromagnetic $\underline{S=1}$ System to Below 1 mK.", Bull. Am. Phys. Soc., 37, 655, (1992).

D. In Progress

1. J. Zhang, J.F. Ma, S.E. Nagler and S.E. Brown, "The Effect of Electric Fields on the CDW $\underline{\text { State". }}$

2. R.F. Shannon, Jr., S.E. Nagler, G.B. Stephenson and J.L. Jordan-Sweet, "Ordering Kinetics in $\mathrm{Cu}_{3} \mathrm{Au}$ at Intermediate Times". 
3. R.F. Shannon, Jr., S.E. Nagler, C.P. Flynn and J. Dura "Ordering Kinetics in Epitaxial Films of Binary Alloys".

4. C.R. Harkless, M.A. Singh, S.E. Nagler, G.B. Stephenson and J.L. Jordan-Sweet, "Small Angle X-ray Scattering Study of Ordering in a Block Copolymer: I: Statics".

5. M.A. Singh, C.R. Harkless, S.E. Nagler, G.B. Stephenson and J.L. Jordan-Sweet, "Small Angle X-ray Scattering Study of ordering in a Block Copolmer: II: Kinetics".

6. D.A. Tennant, T. Perring, S.E. Nagler and S.K. Satija "Observation of the Unbound Spinon Threshold in an Antiferromagnetic Heisenberg Chain".

VI. Travel.

Travel paid for by the DOE Award included several trips to NSLS to perform synchrotron experiments, attendance at the APS March Meeting in 1990, 1991, and 1992, and travel to Gatlinburgh, TN for the DOE discussion meeting on Neutron Scattering in 1990.

Other travel that is related to this work but was paid for from other sources included visiting the Clarendon Laboratories and performing neutron scattering experiments at RAL, and atteudance at the international Conference on Neutron Scattering and the ICM. 
VII. Personnel.

Research Personnel supported by this program during the past 3 years include: $\underline{1989-90}$

S. E. Nagler, P.I.

R. F. Shannon, Jr., Graduate Student.

C. R. Harkless, Graduate Student.

Worked on project but not supported by it:

J. Zhang, Graduate Student.

V. Lane, Graduate Student.

M. Frederickson, Undergraduate Student.

M.A. Singh, Postdoctoral Fellow.

$\underline{1990-91}$

S.E. Nagler, PI.

R.F. Shannon, Jr., Postdoctoral Fellow (partial).

J. Zhang, Graduate Student.

Worked on project but not supported by it:

V. Lane, Graduate Student.

$\underline{1991-92}$

S.E. Nagler, PI.

J.F. Ma, F'ostdoctoral Fellow (partial).

J. Zhang, Graduate Student.

S. Koster, Graduate Student, (partial).

H. Cura, Undergraduate Student.

D. Munini, Undergraduate Student. 


\section{External Support}

Support for $1989-90$

1. U.S. DOE DE-FG05-89ER45280 (this award), $\$ 60,000$

2. NSF Presidential Young Investigator DMR-8553282, S.E. Nagler, PI, $\$ 25,000$

3. DARPA MDA972-88-5-1006, D.B. Tanner, PI, support to S.E. Nagler, $\$ 28,372$.

Support for $1990-91$

1. US DOE DE-FG05-90ER45280 (this award) $\$ 65,000$.

2. NSF Presidential Young Investigator DMR-8553282, S.E. Nagler, PI, $\$ 25,000$.

3. NSF International Program for Co-operative Research US/UK, S.E. Nagler, PI, $\$ 15,000$.

Support for $\underline{1991-92}$

1. US DOE DE-FG05-91ER45280 (this award) $\$ 59,000$.

2. NSF Presidential Young Investigator DMR-8553282, S.E. Nagler, PI, no cost extension.

3. UF Division of Sponsored Research, Research Development Award, S.E. Nagler, PI, ( $\$ 24,393$ for 1 year beginning $5 / 92)$

\section{Figures.}

Figure 1. Speckle pattern of the $\mathrm{Cu}_{3} \mathrm{Au}$ (100) superlattice peak. The Pattern was acquired using a $2 \mathrm{D}$ ccd detector.

Figure 2. CDW $(2 \eta, 0.75+\epsilon,-\eta)$ superlattice peak in blue bronze. (a) After cooling in zero field. (b) After applying a field of $+9 \mathrm{~V} / \mathrm{cm}$. (c) Subsequently applying a field of $-2.3 \mathrm{~V} / \mathrm{cm}$ and waiting 20 minutes. (d) 90 minutes after applying the field of $-2.3 \mathrm{~V} / \mathrm{cm}$. 
Figure 3. Pulsed neutron scattering from $\mathrm{KCuF}_{3}$. The solid line is a fit to a quantum model incorporating a square-root singularity in $S(Q, \omega)$. The dotted line is independently measured nonmagnetic background. 
with light scattering from coherent sources such as lasers. The first experiments to observe speckle in an $\mathrm{x}$-ray diffraction pattern were performed at NSLS beamline $\mathrm{X}-25$, and are described in publication B4 in this report.

In practice, the ability to measure speckle may be quite important for future work on nonequilibrium systems. The speckle arises from collective interference of scattered radiation originating from the entire illuminated volume of the sample. Using a time series

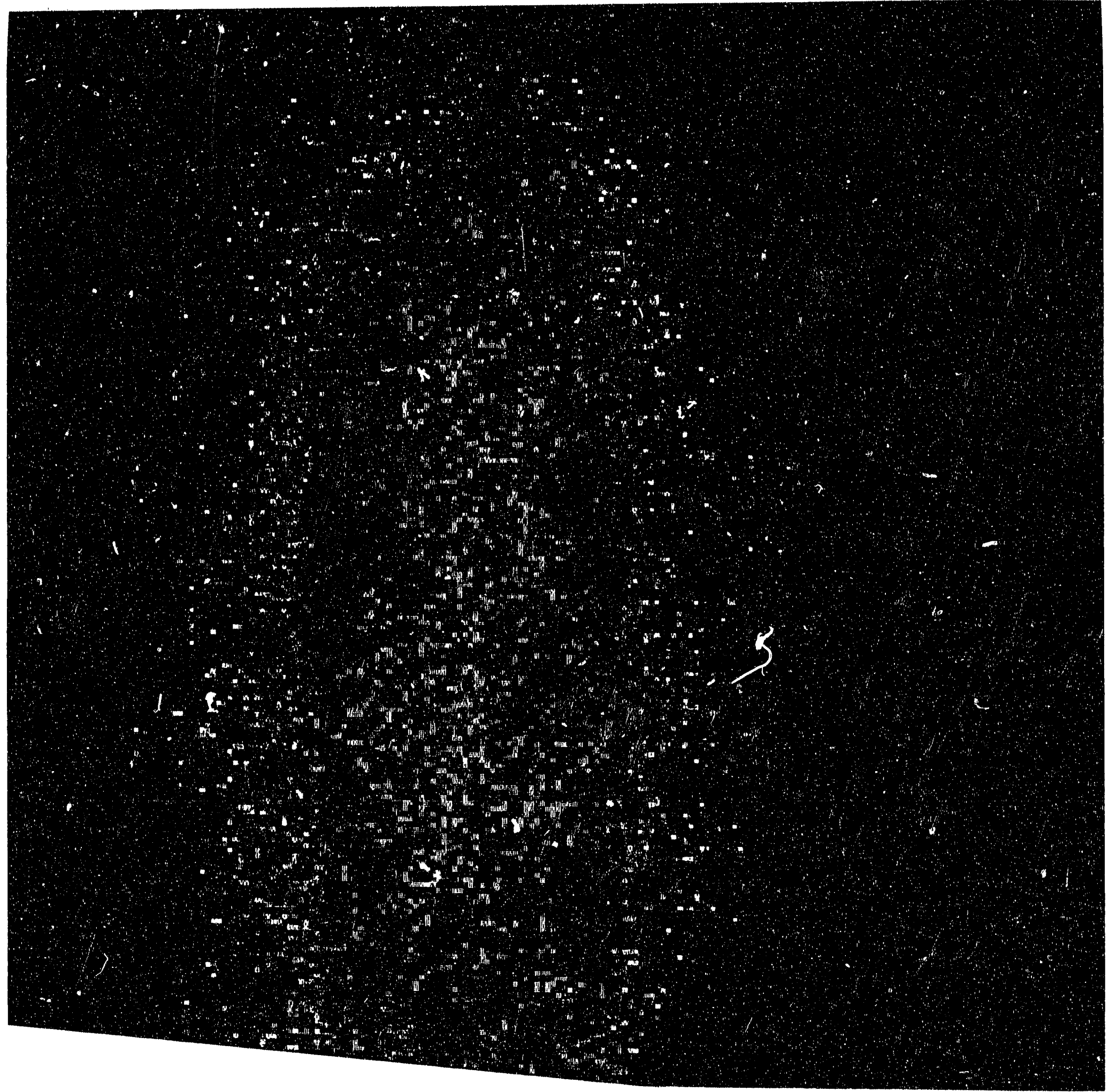




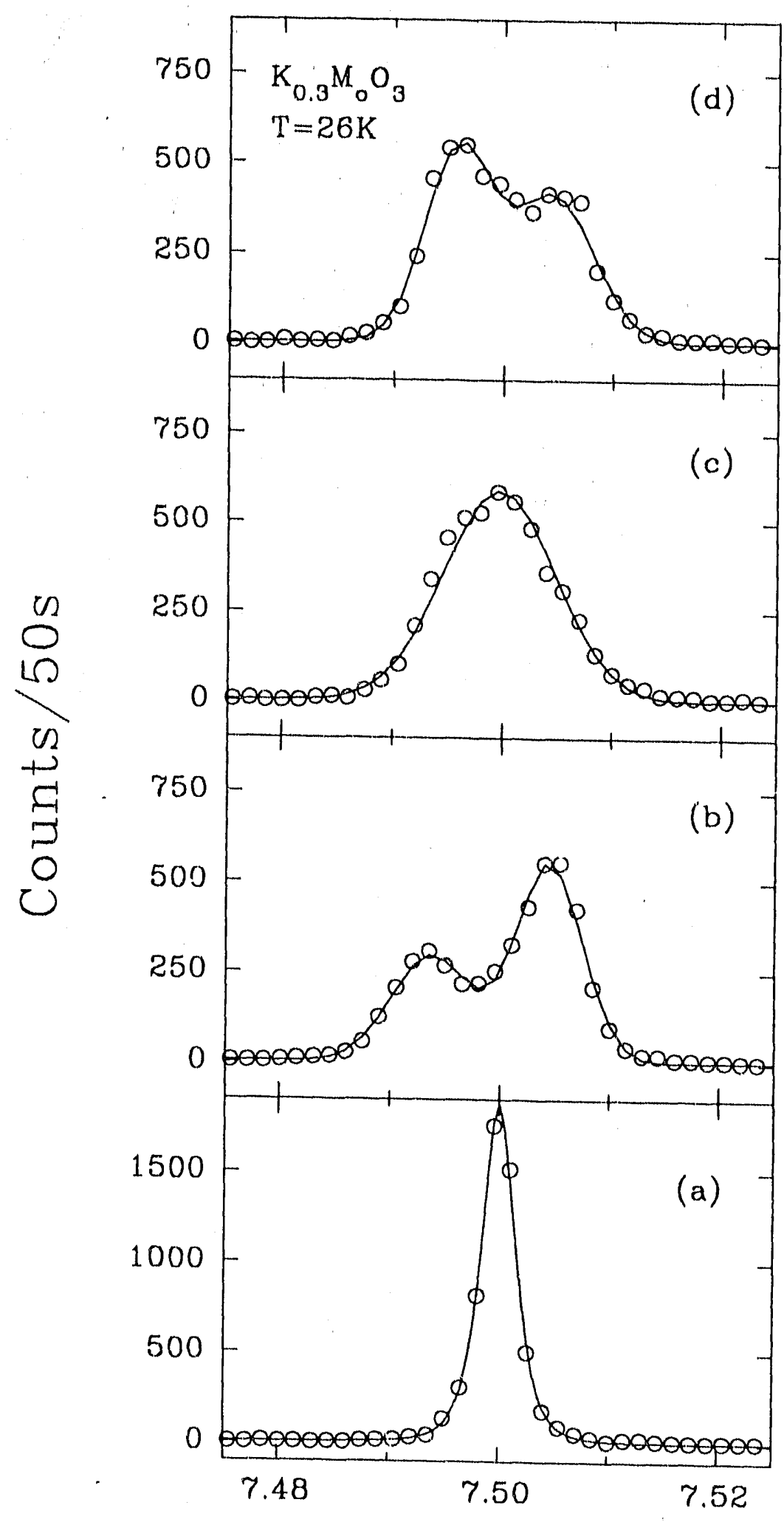

$\eta$ 


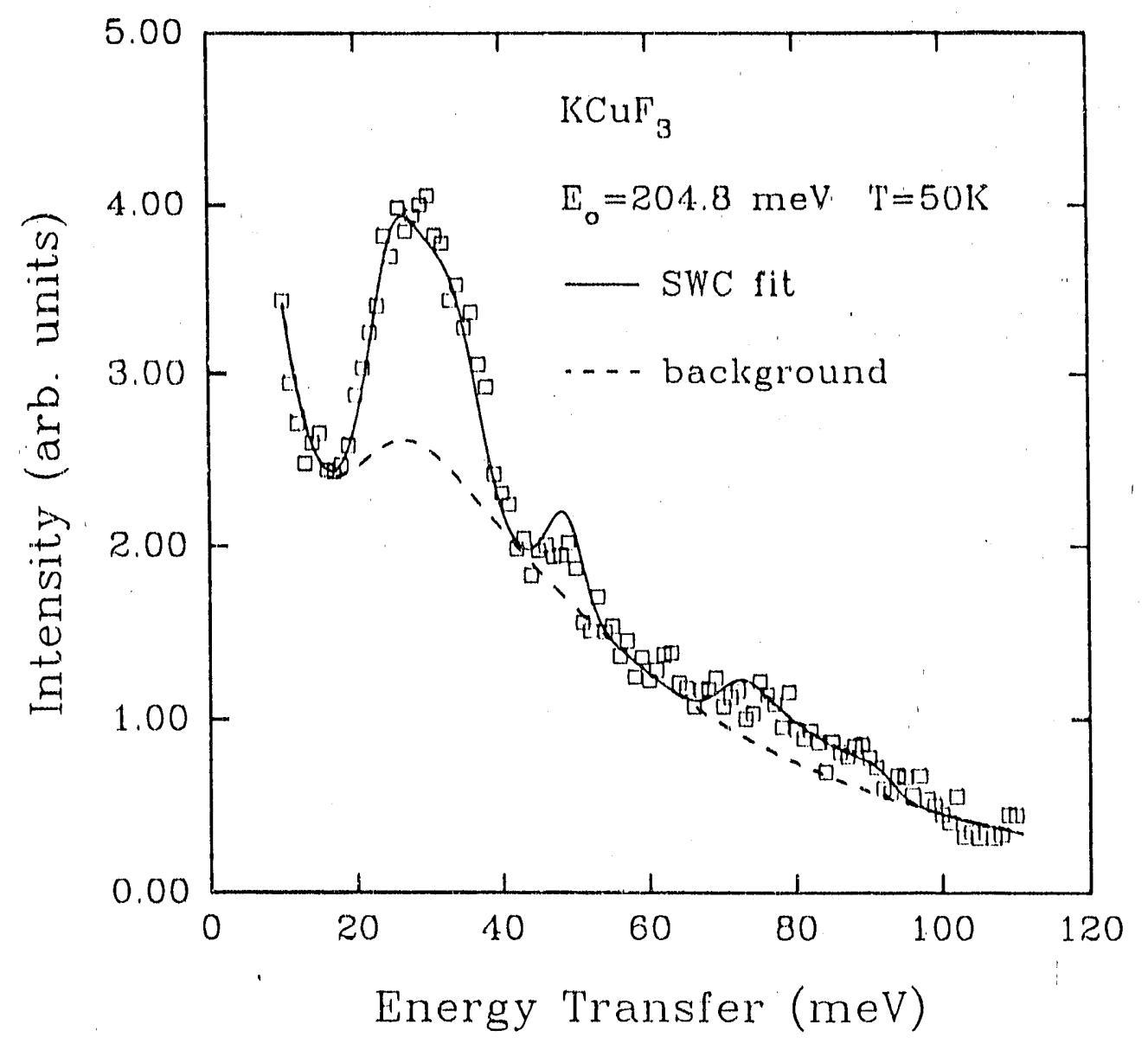

Figun 

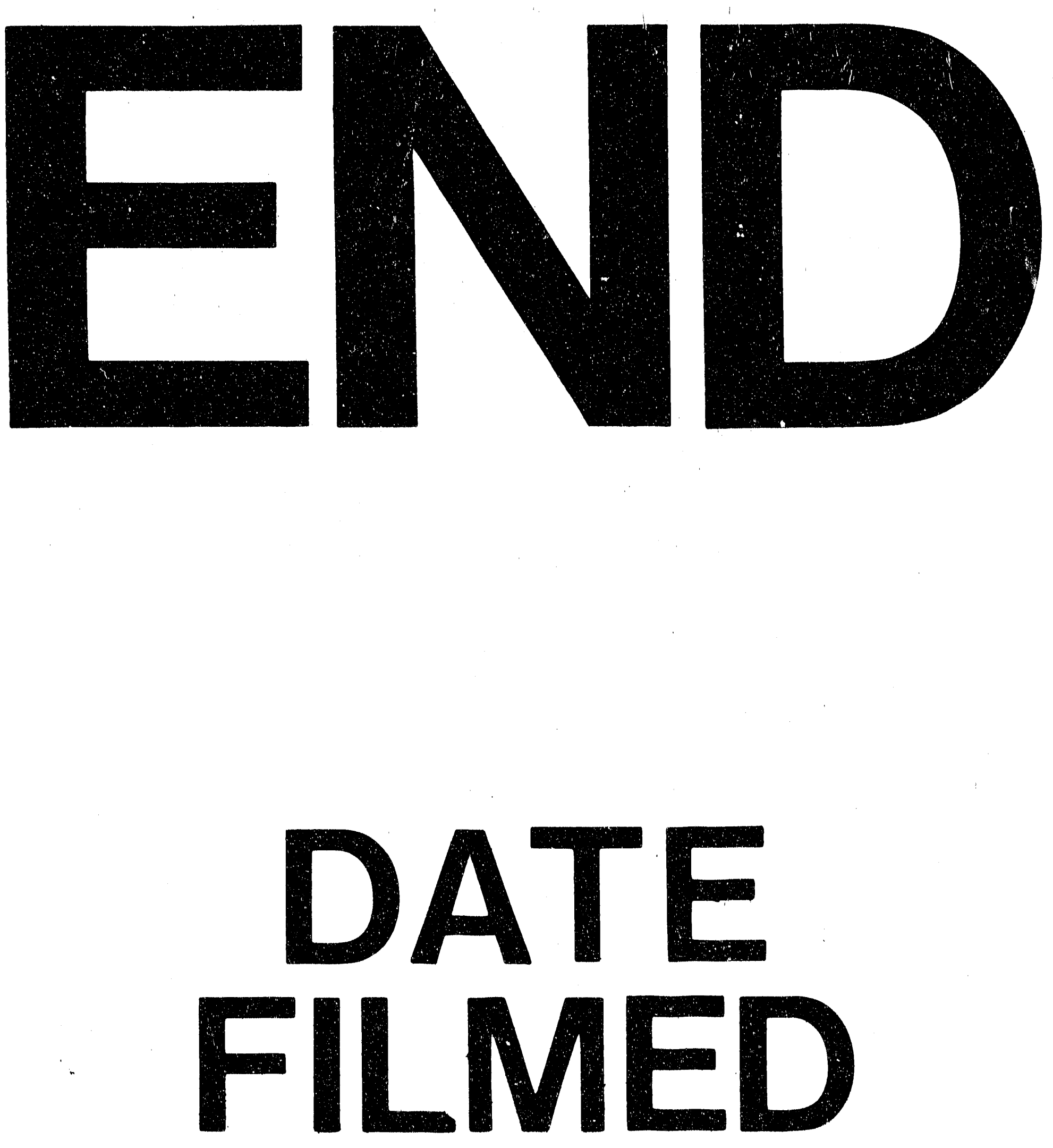

I

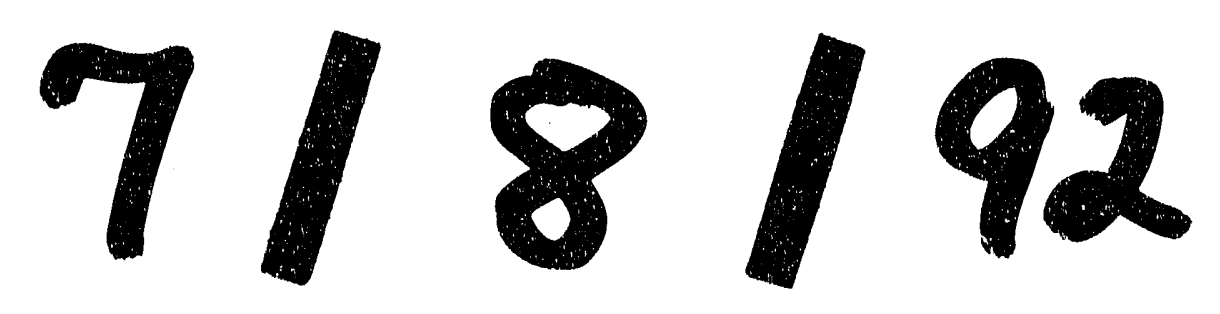


